\title{
PULMONARY TUBERCULOSIS IN CHRONIC KIDNEY DISEASE PATIENTS ON MAINTENANCE HAEMODIALYSIS- A TERTIARY CARE CENTRE'S EXPERIENCE
}

\author{
P. Sharmas Vali1, Kiranmai Ismal2, Manisha Sahay ${ }^{3}$ \\ ${ }_{1}^{1}$ Associate Professor, Department of Nephrology, Osmania General Hospital, Hyderabad, Telangana, India. \\ 2Professor, Department of Nephrology, Osmania General Hospital, Hyderabad, Telangana, India. \\ 3Professor, Department of Nephrology, Osmania General Hospital, Hyderabad, Telangana, India.
}

ABSTRACT
BACKGROUND
Patients of chronic kidney disease on maintenance haemodialysis are many fold prone to manifest active tuberculosis when
compared to the general population. Not only is the timely diagnosis, but also the therapy of tuberculosis can be challenging in
these patients in view of the meticulous dose adjustments.

\section{MATERIALS AND METHODS}

This retrospective study was undertaken with an aim of studying the clinical profile, laboratory characteristics, treatment tolerance and the course of pulmonary tuberculosis in patients of CKD on dialysis. Medical records of chronic kidney disease patients on maintenance haemodialysis for a period ranging from April 2013 to March 2017 were analysed. The diagnosis of Pulmonary Tuberculosis was based on the combination of various tools viz. Sputum microbiology, Nucleic acid testing, Pleural fluid analysis and/or by Radiologic findings.

\section{RESULTS}

A total of forty patients of pulmonary tuberculosis were identified. There was a male preponderance with a male-to-female ratio of 2:1. The most common native kidney disease was chronic glomerulonephritis and the most common risk factor evident was diabetes. Sixty five percent of patients had dialysis vintage ranging from 1-3 years. On tuberculin skin testing, only $45 \%$ of patients had induration of more than $10 \mathrm{~mm}$. All patients received anti-tuberculous therapy at renally modified doses for a total period of 6-9 months and all patients tolerated the therapy well with only one patient experiencing optic neuritis and four patients developing arthralgias. No mortality was recorded in these patients during the study period.

\section{CONCLUSION}

Clinicians need to exercise high index of suspicion to diagnose Tuberculosis in CKD patients. Anti-tuberculous therapy is usually well tolerated in CKD patients, but mandate meticulous dose adjustments.

\section{KEY WORDS}

Tuberculosis, Chronic Kidney Disease, Maintenance Haemodialysis, Anti-Tuberculosis Therapy.

HOW TO CITE THIS ARTICLE: Vali PS, Ismal K, Sahay M. Pulmonary tuberculosis in chronic kidney disease patients on maintenance haemodialysis- a tertiary care centre's experience. J. Evolution Med. Dent. Sci. 2018;7(37):4102-4105, D0I: $10.14260 /$ jemds/2018/917

\section{BACKGROUND}

Tuberculosis (TB) is a common problem in India and spares no set of patient population. India contributes to $23 \%$ of the global TB burden. ${ }^{1}$

By virtue of immunodeficiency, patients of Chronic Kidney Disease (CKD) are prone for Tuberculosis and constitutes an essential subset of TB mandating a particular emphasis concerning identification and treatment. A clinician should bear a high degree of suspicion, while diagnosing Tuberculosis in CKD as many of the clinical manifestations of Tuberculosis can mimic the manifestations of CKD itself. ${ }^{2}$ Not only is the timely diagnosis of Tuberculosis, but also the treatment of Tuberculosis can be challenging in

'Financial or Other Competing Interest': None.

Submission 17-08-2018, Peer Review 28-08-2018,

Acceptance 31-08-2018, Published 10-09-2018.

Corresponding Author:

Dr. P. Sharmas Vali,

Associate Professor,

Department of Nephrology,

Osmania General Hospital,

Hyderabad, Telangana, India.

E-mail: nephron365@gmail.com

DOI: $10.14260 /$ jemds $/ 2018 / 917$ these patients in view of the precise drug dosages, which a clinician should exercise in patients with CKD. It is a wellrecognized fact that Tuberculosis in CKD patients has a predilection for extrapulmonary sites with some studies reporting a prevalence ranging from 38 to $80 \%{ }^{3}$

Besides, CKD patients include a diverse subset of patients with occult or probable TB in whom there would be a robust index of suspicion of Tuberculosis, but with negative workup and who would respond to empirical therapy with antituberculous therapy. Few case series have reported a prevalence of such subset of TB as around 21-50\%.

Further, the tolerability of Anti-Tuberculous Therapy (ATT) is unpredictable in these patients who are independently prone to dyspepsia due to uremia and due to multiple medications, they use in general. This study describes the profile of clinical and laboratory characteristics, treatment tolerance and the course of Pulmonary Tuberculosis in patients of CKD on dialysis (CKD Stage V-D patients).

\section{MATERIALS AND METHODS}

This retrospective study included the patients on maintenance haemodialysis who were diagnosed to have pulmonary tuberculosis. The records of maintenance 
haemodialysis patients from 2013 April to March 2017 were analysed. The inclusion criteria included the patients of CKD Stage V-D who were diagnosed with pulmonary tuberculosis by sputum microbiology, nucleic acid testing, pleural fluid analysis and/ or by radiologic findings. Those patients who had extrapulmonary forms of tuberculosis were ruled out. A total of 40 patients of pulmonary tuberculosis were identified and included in the study. Various parameters such as demographic variables, socioeconomic status, native kidney disease details, risk factors for tuberculosis acquisition, nutritional status, dialysis vintage, nature of access, Arteriovenous Fistula (AVF) failure history, haemodialysis parameters, microbiological and radiologic findings, ATT regimen and its side effects profile were recorded and analysed. IBM SPSS version 22 was used for statistical analysis. Descriptive analysis was carried out by the mean and standard deviation for quantitative variables, frequency and proportion for categorical variables. Data was also represented using appropriate diagrams.

\section{RESULTS}

A total of 40 subjects were included in the final analysis. The mean age was $40.68 \pm 10.39$ with minimal and maximal ages being 18 and 56 respectively ( $95 \%$ CI 37.35 to 44.00 ). Seven (17.50\%) participants were aged upto 30 years, 15 (37.50\%) were aged between 30 and 39 years, $7(17.50 \%)$ were aged between 40 and 49 years and $11(27.50 \%)$ were aged more than 50. Males and females were 27 (67.5\%) and 13 (32.5\%) respectively.

When analysed for Native Kidney Disease (NKD), 9 (22.50\%) participants had Chronic Glomerulonephritis (CGN), 17 (42.50\%) had Chronic Interstitial Nephritis (CIN), 8(20.00\%) had Diabetic Kidney Disease (DKD) and miscellaneous aetiologies in $6(15.00 \%)$. Concerning comorbidities and risk factors for TB $8(20.00 \%)$ participants had diabetic mellitus, 25 (62.50\%) had hypertension, $6(15.00 \%)$ had hypothyroidism and $5(12.50 \%)$ had obesity, while 7 (17.50\%) had chronic steroid usage (Graph 1).

Among the study population, $6(15.00 \%)$ participants were receiving dialysis for $<1$ year, 15 (37.50\%) were receiving dialysis for 1 to 2 years, 11 (27.50\%) were receiving dialysis for 2 to 3 years, $5(12.50 \%)$ were between 3 and 5 years and $3(7.50 \%)$ were receiving dialysis for $>5$ years. All patients had permanent or semi-permanent vascular access with 38 patients (95.00\%) having Arteriovenous Fistula (AVF) access and 2 (5.00\%) had perm catheter. AVF failure was seen in $10(25.00 \%)$ participants. The frequency of dialysis was twice per (week) in 25 (62.50\%) participants and thrice per week in 15 (37.50\%) participants. Average interdialytic weight gain was $<1.5 \mathrm{~kg}$ in $10(25 \%)$ subjects, 1.5 to $2.5 \mathrm{~kg}$ in 15 (37.50\%), 2.5 to $3.5 \mathrm{~kg}$ in $7(17.50 \%)$ and $8(20.00 \%)$ had $>3.5 \mathrm{~kg}$. Among the study population, $10(25.00 \%)$ participants had recurrent fluid overload.

All patients underwent Tuberculin Skin Testing (TST), wherein $6(15.00 \%)$ participants had $<5 \mathrm{~mm}$ of induration, $11(27.50 \%)$ had between 6 and $10 \mathrm{~mm}, 18$ (45.00\%) had between > 11 and $15 \mathrm{~mm}$, and 5 (12.50\%) were > $15 \mathrm{~mm}$. On Radiological imaging (Chest $x$-ray and CT scan chest) infiltrates were noted in $15(37.50 \%)$ participants, $19(47.50 \%)$ had pleural effusion, $4(10.00 \%)$ had cavitary lesions and $2(5.00 \%)$ had other lesions. All patients underwent sputum examination for AFB and 11 (27.50\%) were tested positive. Molecular diagnosis viz. TB PCR test on sputum was positive in $6(15.00 \%)$ participants and 34 (85.00\%) participants were PCR negative (Table 1$)$.

Eleven (27.50\%) participants had $\mathrm{HB}<8,18(45.00 \%)$ had HB between 9 and 10 and 11 (27.50\%) had HB > 10 . Serum albumin level was < 2.5 in 23 (57.5\%), between 2.5 and 3 in 23 (57.5\%), between 3 and 3.5 in 9 (22.5\%), while $>3.5$ in 5 patients. Twelve patients $(30.00 \%)$ had deranged LFT. Serum iPTH was $<70$ in $10(25 \%)$, while the elevation was $>2$ times, 2-4 times and $>4$ times in $14(35 \%), 12(30 \%)$ and $4(10 \%)$. Among the study population, $10(25.00 \%)$ participants were $<70,14(35.00 \%)$ were upto 2 times, 12 $(30.00 \%)$ were between 2 and 4 times and $4(10.00 \%)$ were > 4 times.

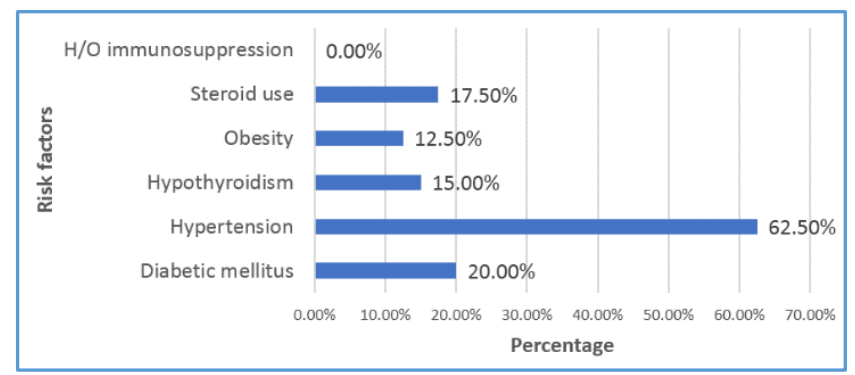

Graph 1. Risk Factors and Comorbidities

\begin{tabular}{|c|c|c|}
\hline $\begin{array}{l}\text { Tuberculosis } \\
\text { Parameters }\end{array}$ & Frequency & Percentage \\
\hline \multicolumn{3}{|c|}{ H/O Contact } \\
\hline Yes & 4 & $10.00 \%$ \\
\hline \multicolumn{3}{|c|}{ Mantoux Test } \\
\hline$<5 \mathrm{~mm}$ & 6 & $15.00 \%$ \\
\hline 6 to $10 \mathrm{~mm}$ & 11 & $27.50 \%$ \\
\hline$>11$ to $15 \mathrm{~mm}$ & 18 & $45.00 \%$ \\
\hline$>15 \mathrm{~mm}$ & 5 & $12.50 \%$ \\
\hline \multicolumn{3}{|c|}{ ESR } \\
\hline Elevated & 30 & $75.00 \%$ \\
\hline Normal & 10 & $25.00 \%$ \\
\hline \multicolumn{3}{|c|}{ Chest X-Ray PA } \\
\hline Infiltrates & 15 & $37.50 \%$ \\
\hline PE & 19 & $47.50 \%$ \\
\hline Cavitary Lesions & 4 & $10.00 \%$ \\
\hline Others & 2 & $5.00 \%$ \\
\hline \multicolumn{3}{|c|}{ Sputum for AFB } \\
\hline Positive & 11 & $27.50 \%$ \\
\hline Negative & 29 & $72.50 \%$ \\
\hline \multicolumn{3}{|c|}{ PCR } \\
\hline Positive & 6 & $15.00 \%$ \\
\hline Negative & 34 & $85.00 \%$ \\
\hline \multicolumn{3}{|c|}{$\begin{array}{l}\text { Table 1. Descriptive analysis of Tuberculosis } \\
\text { Parameters in Study Population }(N=40)\end{array}$} \\
\hline
\end{tabular}

\section{DISCUSSION}

TB affects around 10 million people each year globally and is one of the top ten causes of death worldwide. It is the leading cause of death from a single infectious agent and supersedes HIV/ AIDS. As a whole, only 5-15\% of infected individuals do manifest TB disease in their lifetime. However, such probability of manifesting an overt TB disease is many fold increased in a specific subset of patients such as diabetes, malnutrition, HIV, smoking, alcoholism and chronic kidney disease. ${ }^{5}$ By virtue of their relative immunodeficiency status, CKD patients are prone to CKD by 6-12 times.6,7 
The diagnosis of TB is based on three diagnostic tools viz. rapid molecular tests, sputum smear microscopy and culture based methods.2,8 In addition to these three core tools, imaging will aid in assessing the extent and anatomical pattern of TB. Despite remarkable momentum in diagnostic tool utilisation, only $57 \%$ of TB cases globally are being diagnosed bacteriologically and the rest are being diagnosed clinically. 5

It is worth noting that Tuberculosis is never a diagnosis of based on a single parameter. The task of establishing the diagnosis of TB is based on the combination of clinical, imaging, biochemical, microbiological and histological parameters. ${ }^{9}$ Accordingly, this study has included the conglomeration of all these multiple tools of diagnosis, while labeling a patient as CKD.

There was a male preponderance in this study with a male: female ratio of almost 2: 1 . This is on par with the majority of the studies, which demonstrated similar male preponderance. ${ }^{10,6}$

The evident risk factors in addition to CKD and Dialysis included Diabetes (62.5\%) and Steroid usage (17.5\%). Other comorbidities noted in this study were Hypertension (62.5\%), Hypothyroidism (15\%) and Obesity (12.5\%). A multicentre study from Greece has noted age above 65 years, body mass index, diabetes mellitus, pattern of tuberculin skin test, radiological lesions suggestive of old healed pulmonary tuberculosis and dialysis vintage as risk factors for tuberculosis in chronic haemodialysis patients. ${ }^{11}$

Native Kidney Disease (NKD) might not have a direct influence over the risk of developing tuberculosis. The most common NKD in the current study population was Chronic Glomerulonephritis followed by Chronic Interstitial Nephritis. Even in other studies around the globe, the glomerular disease was the common NKD. 9

Even though diabetes has been mentioned as a common risk factor for the development of tuberculosis in a dialysis patient, that does not translate into the fact that diabetic kidney disease as the leading native kidney disease in these patients. ${ }^{12}$ This could be due to the fact that diabetes reaches a quiescent state by the time the patient of Diabetic Kidney Disease reaches End-Stage Renal Disease (ESRD). ${ }^{9}$

In the current study, almost $65 \%$ of patients had dialysis vintage ranging from 1-3 years. Dialysis vintage was less than one year in $15 \%$ of patients and only $7.5 \%$ of patients were on haemodialysis for more than five years. This was the similar experience from other studies across the globe, and they reported at a peak in the incidence of tuberculosis in patients between 13-36 months of dialysis vintage. ${ }^{9}$

In the current study only $45 \%$ of patients had an induration value of $>10 \mathrm{~mm}$ on Tuberculin Skin Testing (TST), while $15 \%$ of patients had induration of less than 5 $\mathrm{mm}$. All the study subjects underwent only a single tuberculin skin testing. Most experts concur with the opinion that at least two consecutive tests with the double the quantity of Purified Protein Derivative (PPD) would be ideal to enable better interpretation of Tuberculin Skin Testing. ${ }^{13,14}$ TST would be negative in $40-100 \%$ of cases of TB in CKD and dialysis, while paradoxically $6.1-19 \%$ of patients would exhibit TST positivity despite not having a current or historical active tuberculosis. ${ }^{3}$ Similar findings in favour of the non-specific and insensitive nature of TST in dialysis patients were noted in a study conducted at a premier centre in India, wherein authors concluded that the sensitivity was only $20 \%$ and specificity was $9 \%$ for TST in predicting tuberculosis in dialysis patients. ${ }^{15}$

ATT regimen was a standard regimen in all these study subjects and consisted of daily doses of weight-based Isoniazid (INH), Rifampicin (RFM) and alternate-day dosing of full-dose weight-based Ethambutol (ETM) and Pyrazinamide (PZM). The dosages of INH, RFM, ETH and PZM were $5 \mathrm{mg} / \mathrm{kg}, 10 \mathrm{mg} / \mathrm{kg}, 15-25 \mathrm{mg} / \mathrm{kg}$ and $30 \mathrm{mg} / \mathrm{kg}$ respectively. INH and RFM were given on a daily basis, while ETM and PZM were given once on an alternate day. All these patients received renal dose modified Levofloxacin and Pyridoxine $(100 \mathrm{mg} /$ day). The total duration of regimen is variable in these patients from centre to centre. Majority of the centres give for six months to 12 months. $16,3,17$

In the current study, patients received intensive regimen with five drugs for a period of two months to three months and the maintenance phase with three drugs (INH, RFM and Levofloxacin) for a period of 4 to 6 months. The lowest duration of therapy was for six months and the maximum duration was for nine months. On dialysis days, ATT regimen was administered after haemodialysis.

Only one patient developed optic neuritis. Optic neuritis was noticed after 45 days, and it was reversed within a month of stopping of Ethambutol. Four patients developed arthralgias and uric acid was elevated in all these patients. Arthralgias in these patients was attributed to pyrazinamideinduced hyperuricemia. The overall gastrointestinal tolerability was acceptably good with only $20 \%$ reporting dyspeptic symptoms. However, such dyspeptic symptoms were not severe enough to warrant drug withdrawal in any of these patients.

In the current study, all patients completed the specified duration of the ATT regimen and no mortality was recorded in these patients during the course of treatment. All the modern case series reported the similar favourable outcome, while the reported mortality two decades prior was around $22 \% .18,19$ This pattern of favourable outcome could be explained by two reasons. The advancements in the field of early diagnosis of Tuberculosis in all patient subsets including the dialysis patients and the improvement in the nutritional status of dialysis patients.

\section{CONCLUSION}

Tuberculosis has a particular affinity for patients of CKD on dialysis. This infective disease warrants a high index of suspicion on the part of treating clinician to enable diagnosis at the initial stages. The diagnostic tools are akin to those utilised in the general population except for the fact that TST is a less sensitive marker in this subset of patients. The treatment is usually well tolerated, though proper diligence has to be exercised while modulating the dosage and might require a more prolonged therapy in a few. Like in the general population the outcomes of therapy have improved and this favourable development could be attributed to the improvement achieved in arriving at an early diagnosis of TB.

\section{REFERENCES}

[1] Travasso C. India meets target to reduce tuberculosis burden. BMJ 2015;351:h6183. 
[2] Segall L, Covic A. Diagnosis of tuberculosis in dialysis patients: current strategy. Clin J Am Soc Nephrol 2010;5(6):1114-22.

[3] Hussein MM, Mooij JM, Roujouleh H. Tuberculosis and chronic renal disease. Semin Dial 2003;16(1):38-44.

[4] Hussein MM, Bakir N, Roujouleh H. Tuberculosis in patients undergoing maintenance dialysis. Nephrol Dial Transplant 1990;5(8):584-7.

[5] WHO: Global Tuberculosis. 2017. doi:10.1001/jama.2014.11450

[6] Malik GH, Al-Mohaya SA, Al-Harbi AS, et al. Spectrum of tuberculosis in dialysis patients in Saudi Arabia. Saudi J Kidney Dis Transpl 2003;14(2):145-52.

[7] Romanowski K, Clark EG, Levin A, et al. Tuberculosis and chronic kidney disease: an emerging global syndemic. Kidney Int 2016;90(1):34-40.

[8] Richardson RMA. The diagnosis of tuberculosis in dialysis patients. Semin Dial 2012;25(4):419-22.

[9] Erkoc R, Dogan E, Sayarlioglu H, et al. Tuberculosis in dialysis patients, single centre experience from an endemic area. Int J Clin Pract 2004;58(12):1115-7.

[10] Malik GH, Al-Harbi AS, Al-Mohaya S, et al. Eleven years of experience with dialysis associated tuberculosis. Clin Nephrol 2002;58(5):356-62.

[11] Christopoulos AI, Diamantopoulos AA, Dimopoulos PA, et al. Risk factors for tuberculosis in dialysis patients: a prospective multi-center clinical trial. BMC Nephrol 2009;10:36.
[12] Lönnroth K, Roglic G, Harries AD. Improving tuberculosis prevention and care through addressing the global diabetes epidemic: from evidence to policy and practice. Lancet Diabetes Endocrinol 2014;2(9):730-9.

[13] Korzets A, Gafter U. Tuberculosis prophylaxis for the chronically dialysed patient-yes or no? Nephrol Dial Transplant 1999;14(12):2857-9.

[14] Cengiz K, Seker A. Boosted tuberculin skin testing in hemodi- alysis patients. Am J Infect Control 2006;34(6):383-7.

[15] Agarwal S, Gupta S, Bhowmik D, et al. Tuberculin skin test for the diagnosis of latent tuberculosis during renal replacement therapy in an endemic area: a single center study. Indian J Nephrol 2010;20(3):132-6.

[16] Dervisoglu E, Yilmaz A, Sengul E. The spectrum of tuberculosis in dialysis patients. Scand J Infect Dis 2006;38(11-12):1040-4.

[17] Malhotra KK. Treatment of tuberculosis in chronic renal failure, maintenance dialysis and renal transplant. Indian J Nephrology 2003;13:69-71.

[18] Taskapan H, Utas C, Oymak FS, et al. The outcome of tuberculosis in patients on chronic hemodialysis. Clin Nephrol 2000;54(2):134-7.

[19] Venkata RKC, Kumar S, Krishna RP, et al. Tuberculosis in chronic kidney disease. Clin Nephrol 2007;67 (4):217-20. 